\title{
The control parametrization enhancing transform for constrained time-delayed optimal control problems
}

\author{
K. H. Wong* $\quad$ L. S. Jennings ${ }^{\dagger} \quad$ F. Benyah ${ }^{\ddagger}$
}

Received 6 November, 2000; revised 18 April, 2002

*Department of Computational and Applied Mathematics, University of the Witwatersrand, Johannesburg, South AfRICA. mailto:wong@cam.wits.ac.za

${ }^{\dagger}$ Centre for Applied Dynamics and Optimization, Department of Mathematics and Statistics, The University of Western Australia, Crawley, WA 6009, Australia. mailto: les@maths.uwa.edu.au

${ }^{\ddagger}$ Department of Mathematics and Applied Mathematics, University of the Western Cape, Bellville, Cape Town, South AfricA. mailto:fbenyah@uwc.ac.za

${ }^{0}$ See http: / / anziamj . austms.org.au/V43/E043 for this article and ancillary services,

(C) Austral. Mathematical Soc. 2002. Published June 14, 2002. 


\section{Abstract}

The Control Parametrization Enhancing Technique (CPET), is extended to a general class of constrained time-delayed optimal control problems. A model transformation approach is used to convert the time-delayed problem to an optimal control problem involving mixed boundary conditions, but without time-delayed arguments. The CPET is then used to solve this non delayed problem. Two test examples have been solved to illustrate the efficiencies of the CPET for time delayed problems.

\section{Contents}

1 Introduction

2 The Time-Delayed Optimal Control Problem Class

E158

3 Model Transformation

E161

4 The Classical Control Parametrization

E164

5 Control Parametrization Enhancing Transformation

E166

6 Convergence Result

E169

7 Numerical Examples 


\section{Introduction}

A detailed exposition for the control parametrization technique as a basis for solving various constrained optimal control problems numerically in a unified fashion is found in [6]. The classical control parametrization method is a flexible and efficient approach for a large class of optimal control problems. The central idea of the method relies on a simple and elegant approximation mechanism. The time horizon is partitioned into several subintervals and the controls are approximated by piecewise constant (or piecewise linear continuous) functions consistent with the partition. The switching times defined by the partition are referred to as knots, in line with the classification of these approximations as order zero (and one) splines. The heights of the piecewise constant (or linear) functions are now decision variables to be optimized. Control parametrization can thus be used to approximate an optimal control problem by a finite dimensional optimal parameter selection problem.

In $[3,4,7]$, a transformation, to be referred to as the Control Parametrization Enhancing Transform (CPET), was introduced to enhance the classical control parametrization technique. This transform involves the introduction of an additional piecewise constant control function. Using the CPET, the 
switching times are mapped on to an equally spaced set of knots in a new time scale. Hence, the transformed problem can be solved readily and accurately by the usual control parametrization technique. Usually CPET is far superior to the classical control parametrization technique, see [3, 4, 7], and is particularly so for time optimal control problems.

In this paper, the same class of time-delayed optimal control problems as in [2] is considered. A model transformation method similar to that used by Wong [8] is used to convert the time-delayed problem to an optimal control problem involving mixed boundary conditions, but without time-delayed arguments. In this form, the problem can easily be solved using the CPET.

Since the CPET can give rise to a larger dimensional search space than the classical control parametrization method, CPET can produce a better or at least equal sub-optimal cost. Hence all the convergence results which have been proved in [2] for the classical control parametrization method also remain valid for the CPET, when parametrizations are chosen so that the larger dimensional space contains the lower dimensional space.

Two time-delayed optimal control problems are solved by both the classical control parametrization method and the CPET. The results illustrate that the CPET is superior to the classical control parametrization method. 


\section{The Time-Delayed Optimal Control Prob- lem Class}

Consider a process described by the following set of differential equations defined on $(0, T]$;

$$
\dot{\boldsymbol{x}}(t)=\boldsymbol{f}(t, \boldsymbol{x}(t), \boldsymbol{u}(t), \boldsymbol{x}(t-h), \boldsymbol{u}(t-h), \boldsymbol{z})
$$

where $h$ is a positive constant, with the initial conditions and preconditions given by

$$
\begin{aligned}
\boldsymbol{x}(0) & =\boldsymbol{x}^{0}(\boldsymbol{z}), \\
\boldsymbol{x}(t) & =\boldsymbol{\varphi}(t), \quad t \in[-h, 0), \\
\boldsymbol{u}(t) & =\gamma(t), \quad t \in[-h, 0),
\end{aligned}
$$

where $\boldsymbol{x}(t) \in \boldsymbol{R}^{n}, \boldsymbol{u}(t) \in \boldsymbol{R}^{r}$ and $\boldsymbol{z} \in \boldsymbol{R}^{m}$ are respectively the state, the control and system parameter vectors. The vectors $\boldsymbol{f}=\left[f_{1}, \ldots, f_{n}\right]^{\boldsymbol{T}} \in \boldsymbol{R}^{n}$, $\boldsymbol{x}^{0}=\left[x_{1}^{0}, \ldots, x_{n}^{0}\right]^{\boldsymbol{T}} \in \boldsymbol{R}^{n}, \boldsymbol{\varphi}=\left[\varphi_{1}, \ldots, \varphi_{n}\right]^{\boldsymbol{T}} \in \boldsymbol{R}^{n}$, and $\boldsymbol{\gamma}=\left[\gamma_{1}, \ldots, \gamma_{r}\right]^{\boldsymbol{T}} \in$ $\boldsymbol{R}^{r}$ are continuously differentiable with respect to their respective arguments. Because the software system for computing the final optimal control problem will be MISER3 [1, 6] two types of constraints on controls and system parameters are allowable as separate classes of constraints. They are, firstly, system parameter only constraints,

$$
\begin{aligned}
& H_{i}(\boldsymbol{z})=0, \quad k=1, \ldots, N_{1}, \\
& H_{i}(\boldsymbol{z}) \geq 0, \quad k=N_{1}+1, \ldots, N_{2},
\end{aligned}
$$


and all-time linear control only constraints, the latter not used in this paper. All controls and system parameters are subject to upper and lower bounds as follows

$$
\begin{gathered}
\boldsymbol{u}^{L} \leq \boldsymbol{u}(t) \leq \boldsymbol{u}^{U}, \quad \text { for all } t \in[0, T], \\
\boldsymbol{z}^{L} \leq \boldsymbol{z} \leq \boldsymbol{z}^{U} .
\end{gathered}
$$

A measurable function $\boldsymbol{u}:[-h, T] \rightarrow \boldsymbol{R}^{r}$ is called an admissible control if the constraints (4) are satisfied. Let $\mathcal{U}$ be the class of all such admissible controls. Similarly, $\boldsymbol{z} \in \boldsymbol{R}^{m}$ is called an admissible system parameter vector if the constraints (2-3) and (5) are satisfied. Let $\mathcal{Z}$ denote the set of all such admissible system parameter vectors. A pair $(\boldsymbol{u}, \boldsymbol{z}) \in \mathcal{U} \times \mathcal{Z}$ is referred to as an admissible pair. For an admissible pair, let $\boldsymbol{x}(\cdot)$ denote the corresponding solution of the system $(1 \mathrm{a}-1 \mathrm{~d})$.

Constraints involving the state variables or constraints that are nonlinear in control functions are described in the standard canonical form

$$
\begin{aligned}
& G_{k}(\boldsymbol{u}, \boldsymbol{z})=0, \quad k=1, \ldots, N_{3}, \\
& G_{k}(\boldsymbol{u}, \boldsymbol{z}) \geq 0, \quad k=N_{3}+1, \ldots, N_{4},
\end{aligned}
$$

where for each $k=1, \ldots, N_{4}$,

$$
G_{k}(\boldsymbol{u}, \boldsymbol{z})=\Phi_{k}\left(\boldsymbol{x}\left(\tau_{k}\right), \boldsymbol{z}\right)+\int_{0}^{\tau_{k}} \mathcal{L}_{k}(t, \boldsymbol{x}(t), \boldsymbol{u}(t), \boldsymbol{x}(t-h), \boldsymbol{u}(t-h), \boldsymbol{z}) d t .
$$

Here $\Phi_{k}$ and $\mathcal{L}_{k}, k=1, \ldots, N_{4}$, are given real-valued functions which are continuously differentiable with respect to each of their arguments and $\tau_{k} \in$ $(0, T]$ is referred to as the characteristic time for the $k$ th constraint. 
An admissible pair $(\boldsymbol{u}, \boldsymbol{z}) \in \mathcal{U} \times \mathcal{Z}$ is called a feasible pair if the constraints (6) and (7) are satisfied. Let $\mathcal{F}$ be the set of all feasible pairs. The optimal control problem is now formulated as follows:

Problem P: Subject to the dynamical system (1a-1d), find a feasible pair such that the cost functional

$$
G_{0}(\boldsymbol{u}, \boldsymbol{z})=\Phi_{0}(\boldsymbol{x}(T), \boldsymbol{z})+\int_{0}^{T} \mathcal{L}_{0}(t, \boldsymbol{x}(t), \boldsymbol{u}(t), \boldsymbol{x}(t-h), \boldsymbol{u}(t-h), \boldsymbol{z}) d t
$$

is minimized over $\mathcal{F}$. Note the similarity in form of (8) and (9), where $\tau_{0}=T$.

Remark 1 Consider the continuous state inequality constraint defined as follows:

$$
c(t, \boldsymbol{x}(t), \boldsymbol{x}(t-h), \boldsymbol{z}) \geq 0, \quad \text { for all } t \in[0, T] .
$$

Using the $\epsilon-\tau$ method given in [6, Chapter 8], this all-time constraint is approximated by the inequality constraint in canonical form

$$
\tau+\int_{0}^{T} \mathcal{L}_{\epsilon}(c(t, \boldsymbol{x}(t), \boldsymbol{x}(t-h), \boldsymbol{z})) d t \geq 0,
$$

where

$$
\mathcal{L}_{\epsilon}(c)= \begin{cases}c, & \text { if } c<-\epsilon \\ -(c-\epsilon)^{2} / 4 \epsilon, & \text { if }-\epsilon \leq c \leq \epsilon \\ 0, & \text { otherwise }\end{cases}
$$


for all sufficiently small $\epsilon>0$. It is shown in Lemma 8.3.3 of [6] that there exists a $\tau(\epsilon)>0$ such that for each $\tau, 0<\tau<\tau(\epsilon)$, if an admissible pair, $(\boldsymbol{u}, \boldsymbol{z})$, satisfies the constraint (11) it also satisfies the constraint (10). Thus optimal control problems involving all-time state inequality constraints of the form (10) can also be cast in the form of Problem P.

\section{Model Transformation}

Using a method proposed by Wong [8], a time-delayed problem is converted into an optimal control problem involving mixed boundary conditions without time delay. To avoid notational complexity, only a scalar time delay optimal control problem will be considered, so that $n=r=1$. The vector analogue follows easily. Assume that all characteristic times are greater than $h$, the time delay. Let $N$ be an integer such that $(N-1) h<T \leq N h$. Let $\boldsymbol{y}=\left[y_{1}, \ldots, y_{N}\right]^{\boldsymbol{T}}$ and $\boldsymbol{v}=\left[v_{1}, \ldots, v_{N}\right]^{\boldsymbol{T}}$, where $y_{i}(t) \equiv x((i-1) h+t)$ and $\left.v_{i}(t) \equiv u((i-1) h+t)\right)$. For notational convenience, the shortened notation $y_{i}$ for $y_{i}(t)$ and $v_{i}$ for $v_{i}(t)$ will be used. For each $(t, \boldsymbol{y}, \boldsymbol{v}, \boldsymbol{z}) \in[0, h] \times \boldsymbol{R}^{N} \times$ $\boldsymbol{R}^{N} \times \boldsymbol{R}$, let the functions $F_{k}, k=0, \ldots, N_{4}$, and $f_{i}, i=1, \ldots, N$, be 
defined by

$$
\begin{aligned}
& F_{k}(t, \boldsymbol{y}, \boldsymbol{v}, \boldsymbol{z})=\mathcal{L}_{k}\left(t, y_{1}, v_{1}, \varphi(t-h), \gamma(t-h), \boldsymbol{z}\right) \\
& +\sum_{i=2}^{N} \Delta_{k}\left((i-1) h+t, y_{i}, v_{i}, y_{i-1}, v_{i-1}, \boldsymbol{z}\right) \\
& \Delta_{k}(t, p, q, r, s, w)= \begin{cases}\mathcal{L}_{k}(t, p, q, r, s, w), & t \in\left[0, \tau_{k}\right), \\
0, & t \in\left[\tau_{k}, N h\right],\end{cases} \\
& f_{1}(t, \boldsymbol{y}, \boldsymbol{v}, \boldsymbol{z})=f\left(t, y_{1}, v_{1}, \varphi(t-h), \gamma(t-h), \boldsymbol{z}\right), \\
& f_{i}(t, \boldsymbol{y}, \boldsymbol{v}, \boldsymbol{z})=f\left((i-1) h+t, y_{i}, v_{i}, y_{i-1}, v_{i-1}, \boldsymbol{z}\right) \\
& \text { for } i=2, \ldots, N-1 \text {, } \\
& f_{N}(t, \boldsymbol{y}, \boldsymbol{v}, \boldsymbol{z})=\left\{\begin{aligned}
f\left((N-1) h+t, y_{N}, v_{N}, y_{N-1}, v_{N-1}, \boldsymbol{z}\right) & \\
& \text { for } t \in[0, T-(N-1) h], \\
0, \quad & \text { for } t \in(T-(N-1) h, h]
\end{aligned}\right.
\end{aligned}
$$

Let $\overline{\boldsymbol{z}} \in \boldsymbol{R}^{N-1}$ be an additional set of system parameters to be used to equate the new state variables at the initial time point with the appropriate value at the final time point $h$. From the equations $(13 \mathrm{a}-13 \mathrm{~d})$, see that Problem $\mathrm{P}$ is equivalent to the following non-delayed problem:

$$
\underset{\boldsymbol{v}, \boldsymbol{z}, \overline{\boldsymbol{z}}}{\operatorname{minimize}} J(\boldsymbol{v}, \boldsymbol{z}, \overline{\boldsymbol{z}})=\Phi_{0}\left(y_{N}(h), \boldsymbol{z}\right)+\int_{0}^{h} F_{0}(t, \boldsymbol{y}(t), \boldsymbol{v}(t), \boldsymbol{z}) d t
$$


subject to the system

$$
\begin{aligned}
\dot{y}_{i}=f_{i}(t, \boldsymbol{y}(t), \boldsymbol{v}(t), \boldsymbol{z}), & t \in[0, h), \quad i=1, \ldots, N, \\
y_{1}(0)=x^{0}(\boldsymbol{z}), & \\
y_{i+1}(0)=\bar{z}_{i}, & i=1, \ldots, N-1,
\end{aligned}
$$

and the following constraints

$$
\begin{aligned}
H_{i}(\boldsymbol{z})=0, & i=1, \ldots, N_{1}, \\
H_{i}(\boldsymbol{z}) \geq 0, & i=N_{1}+1, \ldots, N_{2}, \\
\boldsymbol{u}^{L} \leq \boldsymbol{v}(t) \leq \boldsymbol{u}^{U}, & \text { for all } t \in[0, h] \\
\boldsymbol{z}^{L} \leq \boldsymbol{z} \leq \boldsymbol{z}^{U}, &
\end{aligned}
$$

and the canonical constraints for $k=1, \ldots, N_{3}$,

$$
G_{k}(\boldsymbol{v}, \boldsymbol{z})=\Phi_{k}\left(y_{\hat{k}}\left(\hat{\tau}_{k}\right), \boldsymbol{z}\right)+\int_{0}^{h} F_{k}(t, \boldsymbol{y}(t), \boldsymbol{v}(t), \boldsymbol{z}) d t=0,
$$

and for $k=N_{3}+1, \ldots, N_{4}$,

$$
G_{k}(\boldsymbol{v}, \boldsymbol{z})=\Phi_{k}\left(y_{\hat{k}}\left(\hat{\tau}_{k}\right), \boldsymbol{z}\right)+\int_{0}^{h} F_{k}(t, \boldsymbol{y}(t), \boldsymbol{v}(t), \boldsymbol{z}) d t \geq 0,
$$

where $\hat{k}$ is the integer such that $(\hat{k}-1) h \leq \tau_{k} \leq \hat{k} h$ and $\hat{\tau}_{k}=\tau_{k}-(\hat{k}-1) h$ and

$$
\bar{G}_{k}(\boldsymbol{v}, \overline{\boldsymbol{z}})=y_{k}(h)-\bar{z}_{k}=0, \quad k=1, \ldots, N-1 .
$$


Remark 2 The constraints (15c) and (22) are equivalent to the fixed boundary conditions, describing continuity of the original states $y_{i+1}(0)=y_{i}(h)$, $i=1, \ldots, N-1$.

\section{The Classical Control Parametrization}

A brief description of the classical control parametrization method is given. Essentially each component $v_{i}(t)$ of the control is approximated by a zeroth order or first order spline defined on a set of fixed knots

$$
\left\{0=t_{0}^{i}, t_{1}^{i}, \ldots, t_{p_{i}}^{i}=h\right\} .
$$

Note that each component may have a different set of knots and that the knots are not necessarily equally spaced. Thus the $i$ th component of the control is

$$
\begin{aligned}
v_{i}(t) & =\sum_{j=1}^{p_{i}} \sigma_{i j} B_{i j}^{0}(t), \\
\text { or } v_{i}(t) & =\sum_{j=0}^{p_{i}} \sigma_{i j} B_{i j}^{1}(t),
\end{aligned}
$$

where $B_{i j}^{0}(t)$ and $B_{i j}^{1}(t)$ are the finite support basis functions for the zeroth order and first order splines respectively. See [1] for further details. After 
control parametrization, the cost function as well as all the constraints can now be regarded as functions of the parameter vector $\boldsymbol{\theta}=(\boldsymbol{\sigma}, \hat{\boldsymbol{z}})$ where

$$
\boldsymbol{\sigma}=\left[\sigma_{11}, \ldots, \sigma_{1, p_{1}}, \sigma_{21}, \ldots, \sigma_{2, p_{2}}, \ldots, \sigma_{N, 1}, \ldots, \sigma_{N, p_{N}}\right]^{\boldsymbol{T}}
$$

and where $\hat{\boldsymbol{z}}=[\boldsymbol{z}, \overline{\boldsymbol{z}}]$. The corresponding problem is

$$
\underset{\boldsymbol{\theta}}{\operatorname{minimize}} G_{0}(\boldsymbol{\theta})
$$

subject to the suitably changed equivalent constraints

$$
\begin{aligned}
H_{i}(\hat{\boldsymbol{z}})=0, & i=1, \ldots, N_{1}, \\
H_{i}(\hat{\boldsymbol{z}}) \geq 0, & i=N_{1}+1, \ldots, N_{2}, \\
G_{k}(\boldsymbol{\theta})=0, & k=1, \ldots, N_{3}, \\
G_{k}(\boldsymbol{\theta}) \geq 0, & k=N_{3}+1, \ldots, N_{4}, \\
u_{i}^{L} \leq \sigma_{i j} \leq u_{i}^{U}, & j=1, \ldots, N, \quad i=1, \ldots, p_{i}, \\
\boldsymbol{z}^{L} \leq \boldsymbol{z} \leq \boldsymbol{z}^{U}, & \\
\bar{G}_{k}(\boldsymbol{\theta})=0, & k=1, \ldots, N-1 .
\end{aligned}
$$

The problem posed by (26-33) is now a standard constrained nonlinear mathematical programming problem solved by means of standard computer codes [5, e.g.]. 


\section{Control Parametrization Enhancing Trans- formation}

Consider the new time scale $s$ which varies from 0 to $M$, where $M$ is an integer. The transformation from $t \in[0, h]$ to $s \in[0, M]$ is defined by the differential equation

$$
\frac{d t(s)}{d s}=w(s)
$$

with the initial condition

$$
t(0)=0,
$$

where the scalar function $w(s)$ is called the enhancing control. It is a piecewise constant function (usually) with possible discontinuities at the pre-fixed knots $s=0,1,2, \ldots, M$. That is,

$$
w(s)=\sum_{i=1}^{M} w_{i} \chi_{i}(s),
$$

where $\chi_{i}(s)$ is the indicator function defined by

$$
\chi_{i}(s)= \begin{cases}1, & \text { if } s \in[i-1, i), \\ 0, & \text { otherwise }\end{cases}
$$

Integrating (34a)

$$
t(s)=\int_{0}^{s} w(\tau) d \tau=\sum_{j=1}^{i-1} w_{j}+w_{i}(s-(i-1)), \quad s \in[i-1, i] .
$$


The optimal control function is now written in terms of the new time variable $s$. Let $\hat{\boldsymbol{v}}(s)=\boldsymbol{v}(t(s)), \hat{\boldsymbol{y}}(s)=\boldsymbol{y}(t(s))$ and $\hat{\boldsymbol{z}}=(\boldsymbol{z}, \overline{\boldsymbol{z}})$, then

$$
\hat{\boldsymbol{x}}(s)=\left[\hat{\boldsymbol{y}}(s)^{\boldsymbol{T}}, t(s)\right]^{\boldsymbol{T}}
$$

are the new state variables, and

$$
\hat{\boldsymbol{u}}(s)=\left[\hat{\boldsymbol{v}}(s)^{\boldsymbol{T}}, w(s)\right]^{\boldsymbol{T}}
$$

are the new control variables. The equivalent transformed optimal control problem is re-stated as

$$
\underset{\hat{\boldsymbol{u}}, \hat{z}}{\operatorname{minimize}} G_{0}(\hat{\boldsymbol{u}}, \hat{\boldsymbol{z}})=\Phi_{0}(\hat{\boldsymbol{y}}(M), \boldsymbol{z})+\int_{0}^{M} w(s) F_{0}(t(s), \hat{\boldsymbol{y}}(s), \hat{\boldsymbol{v}}(s), \boldsymbol{z}) d s,
$$

subject to the dynamical system

$$
\frac{d \hat{\boldsymbol{x}}(s)}{d s}=\left[\begin{array}{c}
w(s) f(t(s), \hat{\boldsymbol{y}}(s), \hat{\boldsymbol{v}}(s), \boldsymbol{z}) \\
w(s)
\end{array}\right], \quad s \in[0, M],
$$

with initial conditions

$$
\begin{aligned}
& \hat{y}_{1}(0)=x^{0}(\boldsymbol{z}), \\
& \hat{y}_{i+1}(0)=\bar{z}_{i}, \quad i=1, \ldots, N-1, \\
& t(0)=0,
\end{aligned}
$$


and the following constraints

$$
\begin{aligned}
H_{i}(\boldsymbol{z})=0, & i=1, \ldots, N_{1}, \\
H_{i}(\boldsymbol{z}) \geq 0, & i=N_{1}+1, \ldots, N_{2}, \\
\boldsymbol{u}^{L} \leq \hat{\boldsymbol{v}}(s) \leq \boldsymbol{u}^{U}, & \text { for all } s \in[0, M] \\
\boldsymbol{z}^{L} \leq \boldsymbol{z} \leq \boldsymbol{z}^{U}, &
\end{aligned}
$$

and the canonical constraints

$$
\begin{array}{ll}
G_{k}(\hat{\boldsymbol{u}}, \boldsymbol{z})=0, & k=1, \ldots, N_{3}, \\
G_{k}(\hat{\boldsymbol{u}}, \boldsymbol{z}) \geq 0, & k=N_{3}+1, \ldots, N_{4},
\end{array}
$$

where

$$
\begin{aligned}
G_{k}(\hat{\boldsymbol{u}}, \boldsymbol{z}) & \left.=\Phi_{k}\left(\hat{y}_{\hat{k}}\left(M_{k}\right), \boldsymbol{z}\right)\right)+\int_{0}^{M_{k}} w(s) F_{k}(t(s), \hat{\boldsymbol{y}}(s), \hat{\boldsymbol{v}}(s), \boldsymbol{z}) d s \\
\bar{G}_{k}(\hat{\boldsymbol{u}}, \overline{\boldsymbol{z}}) & =\hat{y}_{k}(h)-\bar{z}_{k}=0, \quad k=1, \ldots, N-1 \\
\hat{G}(\hat{\boldsymbol{u}}) & =t(M)=h
\end{aligned}
$$

Here $M_{k}=s\left(\hat{\tau}_{k}\right)$ is one of the knot points in $[0,1]$. For the new control $w(s)$ it is necessary that $w(s) \geq 0, s \in[0, M]$, so that the new state $t(s)$ is monotonic non-decreasing on $[0, h]$. Appropriate bounds on the enhancing control values ensure this.

Remark 3 As mentioned in [7], in the transformed problem, only the knots contribute to the discontinuities of the state differential equations. Thus, all 
locations of the discontinuities of the state differential equations are known and fixed during the optimization process. These locations will not change from one iteration to the next during the optimization process.

\section{Convergence Result}

The basic idea behind the CPET method developed in [7] aims to include the switching times as parameters to be optimized and at the same time to avoid the numerical difficulties mentioned in [7]. The CPET method captures the discontinuities of the optimal control problem if the number of knots in the partition of the new time horizon is greater than or equal to the number of discontinuities of the optimal control.

Since the time-delayed problem considered in this paper has almost the same structure as that considered in [2], all the convergence results of the classical control parametrization method obtained in [2] remain valid, provided we make the same assumptions as those given in (A1-A8) of [2]. Since the enhancing parameters are allowed to vary, the control parametrization enhancing technique gives rise to a larger search space and hence produces a better or at least equal sub-optimal cost. Hence, by the squeeze theorem, the convergence of the problem with an enhancing control is guaranteed. Thus all the convergence results in [2] remain valid for the CPET problem. Of course the existence of local optima mean that computed solutions may bear no resemblance to the solutions of the convergence results. 


\section{Numerical Examples}

The following example is a standard simple example used to test algorithms for delay equations and is Example 7.1 of [2].

Example 4

$$
\text { minimize } G_{0}(u)=x(2)^{2}+\int_{0}^{2}\left[x(t)^{2}+u(t)^{2}\right] d t,
$$

subject to the delay differential equation

$$
\begin{array}{lrl}
\dot{x}(t) & =x(t) \sin (x(t))+x(t-1)+u(t), & t \in[0,2], \\
x(t) & =10, & t \in[-1,0],
\end{array}
$$

together with the terminal state equality constraint

$$
\Phi_{1}(x(2))=-119.854+22 x(2)-x(2)^{2}=0,
$$

and the continuous state inequality constraint

$$
g_{1}(t, x(t))=114.6-8 t-x(t)^{2} \geq 0, \quad t \in[0,2] .
$$

Classical Control Parametrization Technique Formulation: The following transformations re-formulate Example 4 into a nondelay problem to be solved by a Classical Control Parametrization Method, on the interval $[0,1]$ : 


\section{Transformation 1:}

$$
\begin{array}{llrl}
y_{1}(t) & =x(t), & & t \in[0,1], \\
y_{2}(t) & =x(t+1), & & t \in[0,1], \\
v_{1}(t) & =u(t), & & t \in[0,1], \\
v_{2}(t) & =u(t+1), & & t \in[0,1] .
\end{array}
$$

The resulting non-delayed re-formulation of Example 4 is as follows:

$$
\operatorname{minimize} G_{0}(u)=\left(y_{2}(1)\right)^{2}+\int_{0}^{1}\left[y_{1}(t)^{2}+y_{2}(t)^{2}+u_{1}(t)^{2}+u_{2}(t)^{2}\right] d t,
$$

subject to the non-delay differential equations on the interval $[0,1]$

$$
\begin{array}{lr}
\dot{y}_{1}(t)=y_{1}(t) \sin \left(y_{1}(t)\right)+10+u_{1}(t), & y_{1}(0)=10, \\
\dot{y}_{2}(t)=y_{2}(t) \sin \left(y_{2}(t)\right)+y_{1}(t)+u_{2}(t), & y_{2}(0)=z,
\end{array}
$$

subject to all-time inequality constraints

$$
\begin{array}{ll}
114.6-8 t-y_{1}^{2}(t) \geq 0, & t \in[0,1], \\
106.6-8 t-y_{2}^{2}(t) \geq 0, & t \in[0,1],
\end{array}
$$

together with the terminal state equality constraint

$$
\begin{aligned}
\bar{G}_{1}(\boldsymbol{u}, \boldsymbol{z}) & =y_{1}(1)-z=0, \\
\Phi_{1}\left(y_{2}(1)\right) & =-119.854+22 y_{2}(1)-y_{2}(1)^{2}=0 .
\end{aligned}
$$




\section{The Control Enhancement Parametrization Technique Formula-}

tion: Transformation 1, with a change of scale, re-formulates Example 4 to be solved using the Control Parametrization Enhancement Technique, on the interval $[0,5]$ :

$$
\operatorname{minimize} J(v)=y_{2}(5)^{2}+\int_{0}^{5} v_{3}(t)\left[y_{1}(t)^{2}+y_{2}(t)^{2}+v_{1}(t)^{2}+v_{2}(t)^{2}\right] d t,
$$

subject to the nondelay differential equations on the interval $[0,5]$

$$
\begin{array}{llrl}
\dot{y}_{1}(t) & =v_{3}(t)\left(y_{1}(t) \sin \left(y_{1}(t)\right)+10+v_{1}(t)\right), & & y_{1}(0)=10, \\
\dot{y}_{2}(t) & =v_{3}(t)\left(y_{2}(t) \sin \left(y_{2}(t)\right)+y_{1}(t)+v_{2}(t)\right), & & y_{2}(0)=z, \\
\dot{y}_{3}(t) & =v_{3}(t), & & y_{3}(0)=0,
\end{array}
$$

subject to all-time inequality constraints

$$
114.6-8 y_{3}(t)-y_{1}^{2}(t) \geq 0, \quad 106.6-8 y_{3}(t)-y_{2}^{2}(t) \geq 0,
$$

together with the terminal state equality constraints

$$
y_{1}(5)=z, \quad y_{3}(5)=1, \quad-119.854+22 y_{2}(5)-y_{2}^{2}(5)=0 .
$$

Solving the non-delayed problem by the classical control parametrization method with 8 piecewise constant control parameters on the interval $[0,1]$ gives a minimum value of 331.63. On the other hand, solving the nondelayed problem by the CPET with 5 piecewise constant control parameters 


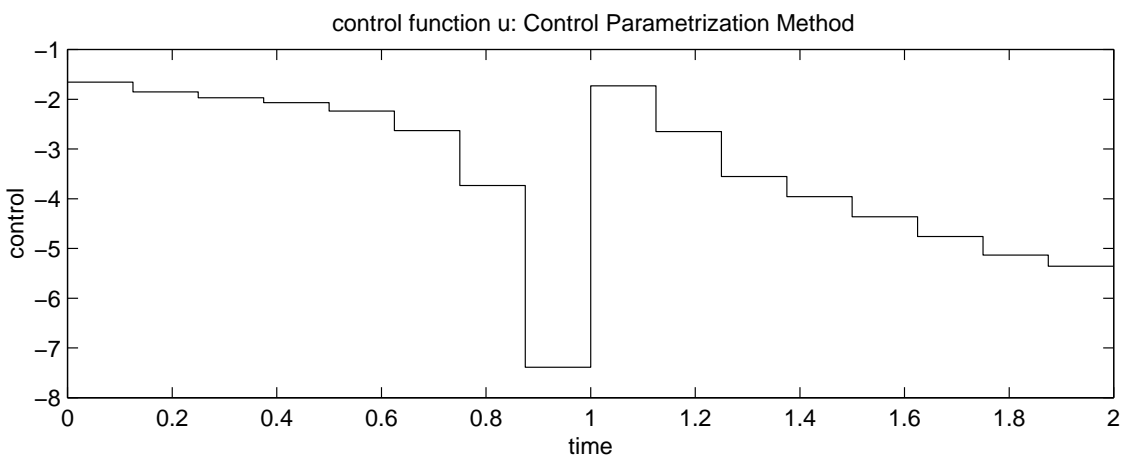

state function $\mathrm{x}$ : Control Parametrization Method

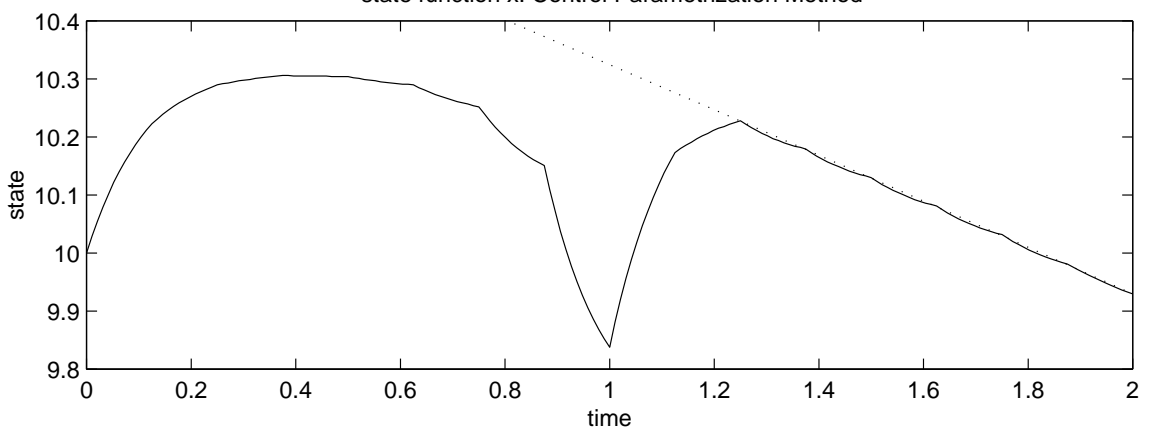

Figure 1: Example 4-Piecewise constant solutions, control parametrization method. 

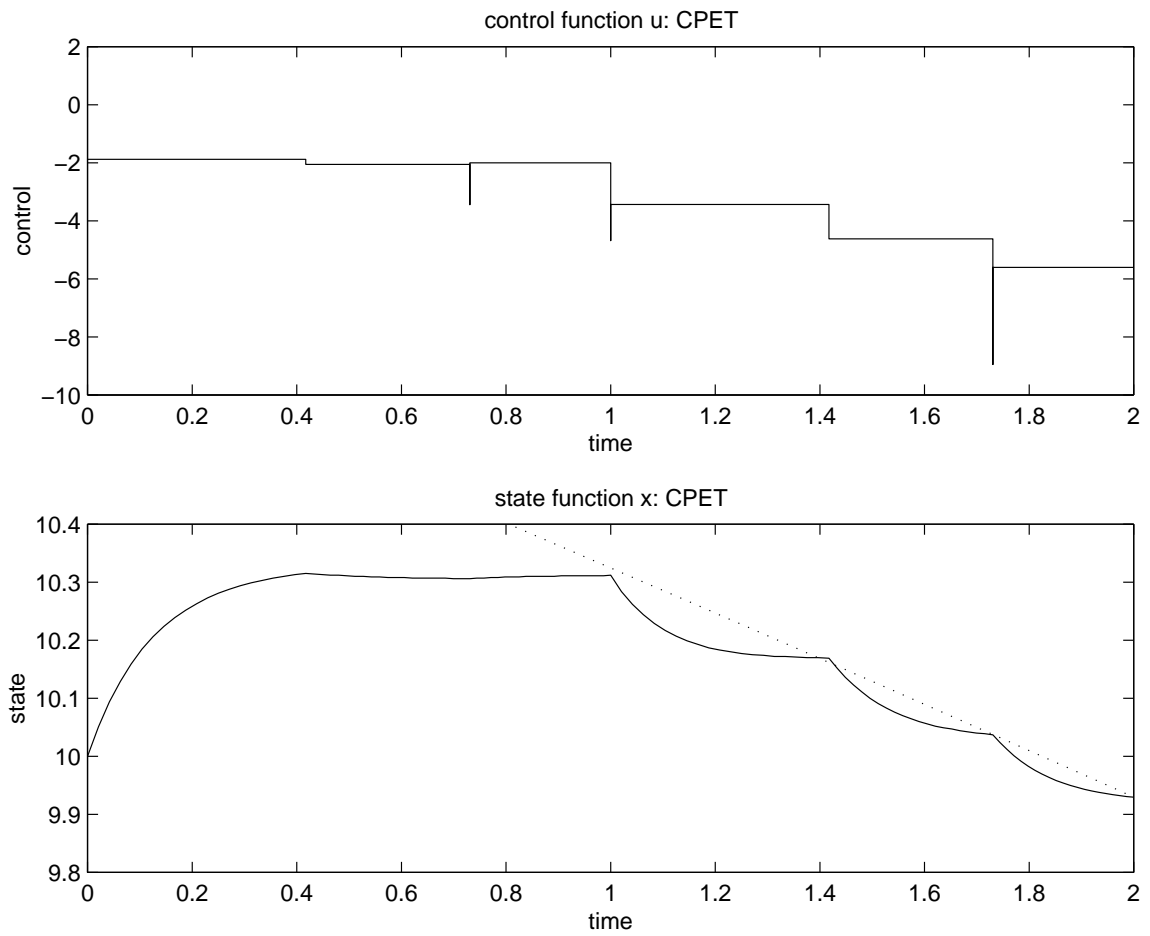

Figure 2: Example 4-Piecewise constant solutions, CPET. 


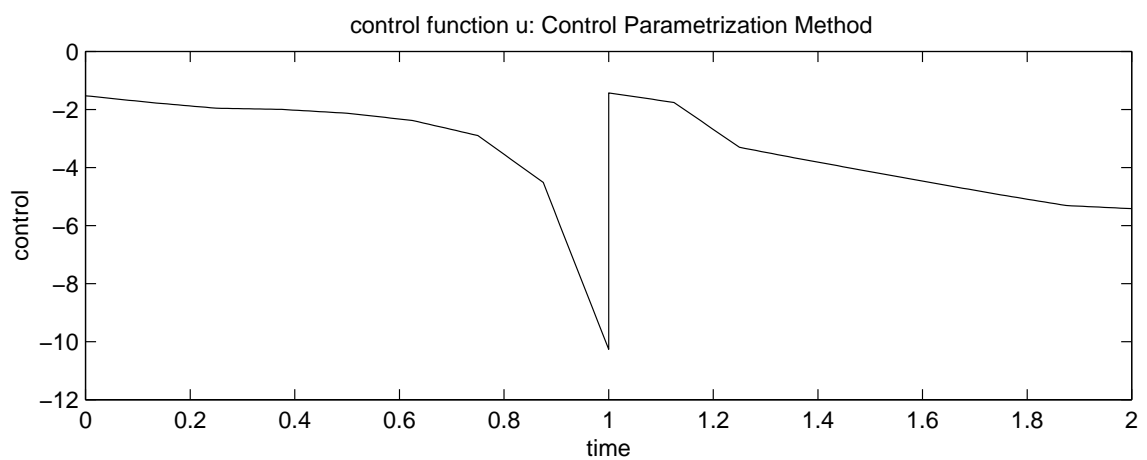

state function $\mathrm{x}$ : Control Parametrization Method

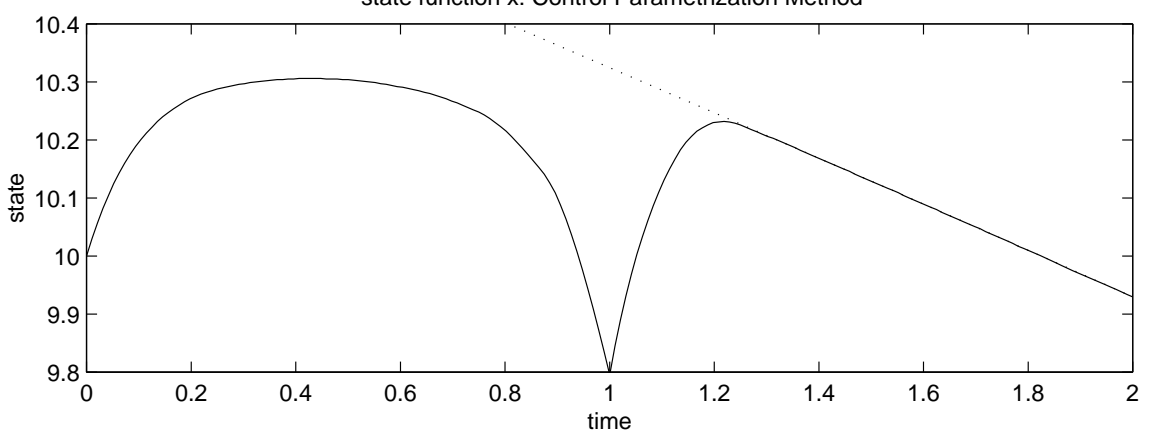

Figure 3: Example 4-Piecewise linear solutions, control parametrization method. 

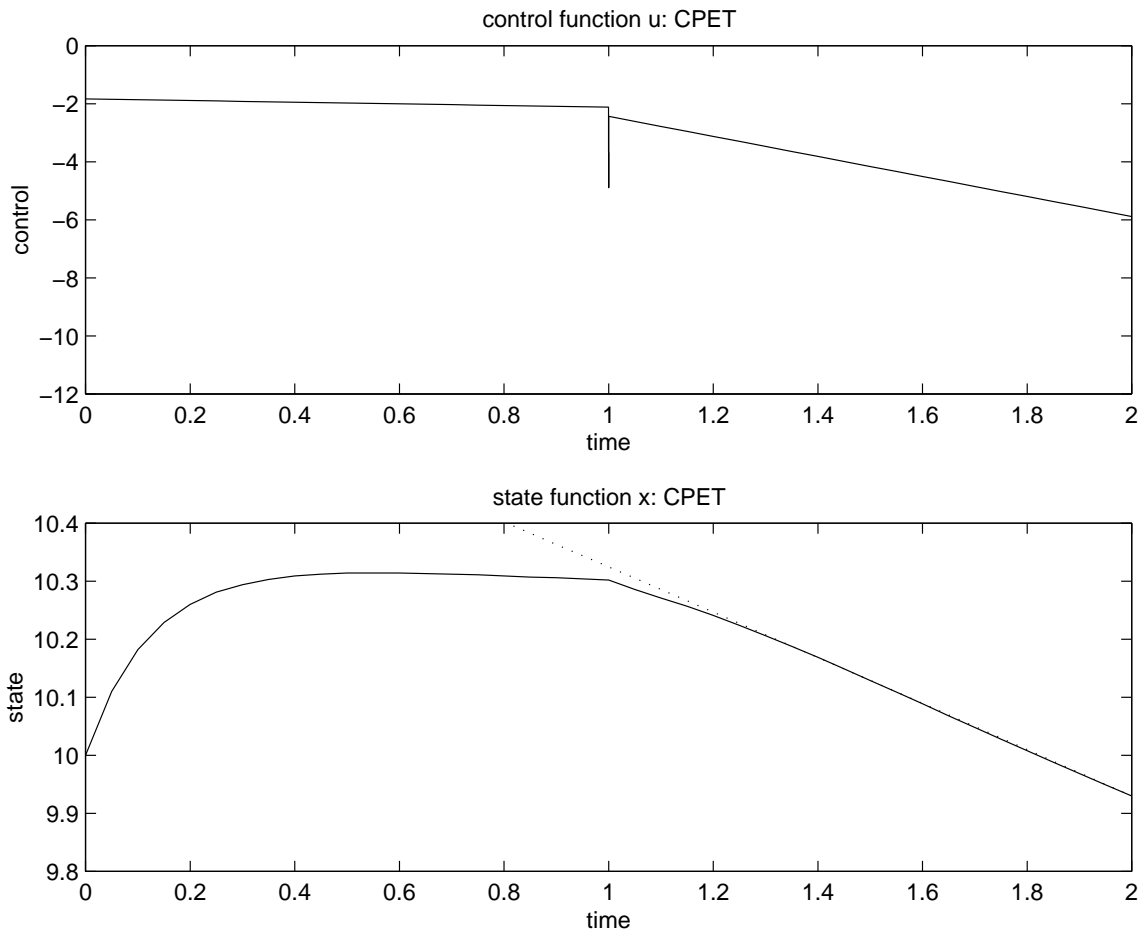

Figure 4: Example 4-Piecewise linear solutions, CPET. 
on the interval $[0,1]$ gives a minimum value of 330.12 . Using piecewise linear controls the corresponding values are 331.08 and 328.93 respectively. Thus, the CPET is superior to the classical control parametrization method. Note that the CPET computation has effectively used very few control parameters compared to the non CPET computation, three for piecewise constant and two for piecewise linear, and has a better objective value in both cases. The vertical lines on the CPET control function indicate a function value on a set of (computationally) measure zero. Notice also that if it was necessary for the control to be continuous, then more constraints have to be used to do this at the time points which are multiples of $h$. This is difficult if some intervals become redundant as seen in Figures 3-4. The dotted line is the all-time constraint.

In view of the above results, it would be worthwhile to recompute using a smaller number of control parameters, especially in the piecewise linear case which seems to indicate that CPET is not necessary. No doubt local minima are playing a role in the above computation.

Example 5 The following example is an adaption of a standard difficult problem used to test the classical parametrization technique and is Example 7.2 from [2].

$$
\operatorname{minimize} G_{0}(u)=\int_{0}^{1}\left[x_{1}(t)^{2}+x_{2}(t)^{2}+0.005 u(t)^{2}\right] d t,
$$


subject to the delay differential equations, with initial conditions

$$
\begin{array}{lrl}
\dot{x}_{1}(t) & =x_{2}(t-1 / 3), & x_{1}(0)=0, \\
\dot{x}_{2}(t)=-x_{2}(t)+u(t), & x_{2}(0)=-1,
\end{array}
$$

and pre-conditions

$$
x_{2}(t)=-1, t \in[-1 / 3,0],
$$

together with the terminal state inequality constraint

$$
\Phi_{1}(\boldsymbol{x}(1))=-x_{2}(1) \geq 0,
$$

and the continuous state inequality constraint

$$
g_{1}(t, \boldsymbol{x}(t))=-x_{2}(t)+8(t-0.5)^{2}-0.5 \geq 0, \quad t \in[0,1] .
$$

Classical Control Parametrization Technique Formulation:

The following transformations re-formulate Example 5 into a nondelay problem to be solved by a Classical Control Parametrization Method, on the interval $[0,1 / 3]$ : 


\section{Transformation 2:}

$$
\begin{array}{llrl}
y_{1}(t) & =x_{1}(t), & t \in[0,1 / 3] \\
y_{2}(t) & =x_{1}(t+1 / 3), & t \in[0,1 / 3] \\
y_{3}(t)=x_{1}(t+2 / 3), & t \in[0,1 / 3] \\
y_{4}(t)=x_{2}(t), & t \in[0,1 / 3] \\
y_{5}(t)=x_{2}(t+1 / 3), & t \in[0,1 / 3] \\
y_{6}(t)=x_{2}(t+2 / 3), & t \in[0,1 / 3] \\
v_{1}(t)=u(t), & t \in[0,1 / 3] \\
v_{2}(t)=u(t+1 / 3), & t \in[0,1 / 3] \\
v_{3}(t)=u(t+2 / 3), & t \in[0,1 / 3]
\end{array}
$$

The resulting non-delayed re-formulation of Example 5 is as follows:

$$
\begin{array}{r}
\operatorname{minimize} G_{0}(\boldsymbol{v})=\int_{0}^{1 / 3}\left[y_{1}(t)^{2}+y_{2}(t)^{2}+y_{3}(t)^{2}+y_{4}(t)^{2}+y_{5}(t)^{2}+y_{6}(t)^{2}\right. \\
\left.+0.005\left(v_{1}(t)^{2}+v_{2}(t)^{2}+v_{3}(t)^{2}\right)\right] d t
\end{array}
$$


subject to the nondelay differential equations on the interval $[0,1 / 3]$

$$
\begin{array}{lrl}
\dot{y}_{1}(t) & =-1, & y_{1}(0)=0, \\
\dot{y}_{2}(t) & =y_{4}(t), & y_{2}(0)=z_{1}, \\
\dot{y}_{3}(t)=y_{5}(t), & y_{3}(0)=z_{2}, \\
\dot{y}_{4}(t)=-y_{4}(t)+v_{1}(t), & y_{4}(0)=-1, \\
\dot{y}_{5}(t)=-y_{5}(t)+v_{2}(t), & y_{5}(0)=z_{3}, \\
\dot{y}_{6}(t)=-y_{6}(t)+v_{3}(t), & y_{6}(0)=z_{4},
\end{array}
$$

subject to the continuous state inequality constraint

$$
\begin{gathered}
g_{1}(t, \boldsymbol{y}(t))=8(t-0.5)^{2}-0.5-y_{4}(t) \geq 0, \\
g_{2}(t, \boldsymbol{y}(t))=8(t-1 / 6)^{2}-0.5-y_{5}(t) \geq 0, \\
g_{3}(t, \boldsymbol{y}(t))=8(t+1 / 6)^{2}-0.5-y_{6}(t) \geq 0
\end{gathered}
$$

together with the terminal state equality constraints

$$
\begin{aligned}
\bar{G}_{1}(\boldsymbol{v}, \boldsymbol{z}) & =y_{1}(1 / 3)-z_{1}=0, \\
\bar{G}_{2}(\boldsymbol{v}, \boldsymbol{z}) & =y_{2}(1 / 3)-z_{2}=0, \\
\bar{G}_{3}(\boldsymbol{v}, \boldsymbol{z}) & =y_{4}(1 / 3)-z_{3}=0, \\
\bar{G}_{4}(\boldsymbol{v}, \boldsymbol{z}) & =y_{5}(1 / 3)-z_{4}=0,
\end{aligned}
$$

and subject to the terminal state inequality constraint

$$
\Phi_{1}(\boldsymbol{y}(1 / 3))=-y_{6}(1 / 3) \geq 0
$$


Solving the non-delayed problem by the classical control parametrization method with 4 piecewise constant control parameters on the interval $[0,1 / 3]$ gives a minimum value of 0.283495511 . On the other hand, solving the nondelayed problem by the CPET with 4 piecewise constant control parameters on the interval $[0,1 / 3]$ gives a minimum value of 0.280028250 . Once again, see the CPET is superior to the classical control parametrization method. Using piecewise linear controls makes it possible to follow the quadratic exactly.

\section{Conclusions}

Optimal control problems with delay differential equations and a fixed time horizon have been shown to be computable more efficiently with the CPET algorithm than the standard control parametrization method. For a small delay, in comparison to the time horizon, the method of [2] creates a large number of new state variables, with attendant boundary value constraints. This disadvantage is tempered somewhat by the existence of MISER3 as a computational aid to readily compute solutions to constrained optimal control problems. Variable time delay problems, where the time delay depends on time or state, still presents an interesting challenge. 

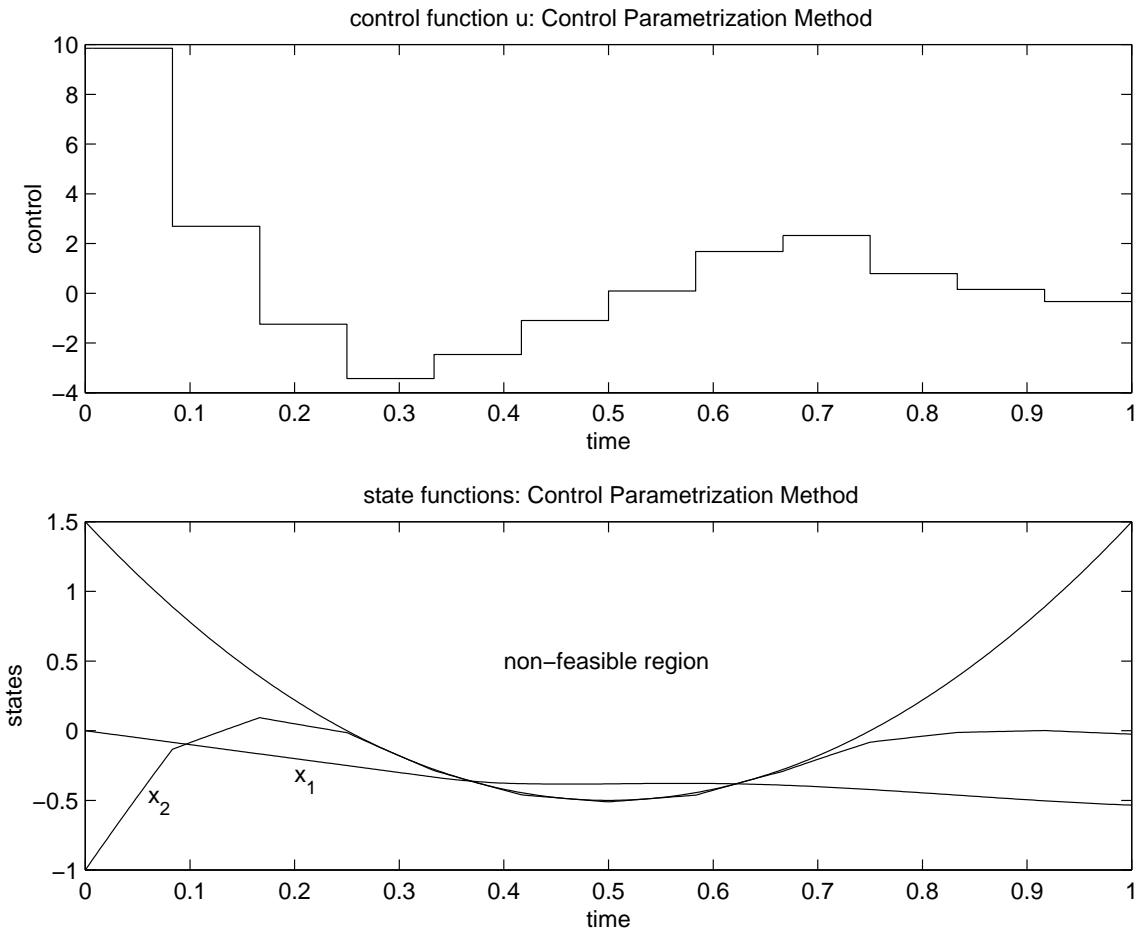

Figure 5: Example 5-Solution by Standard Method 

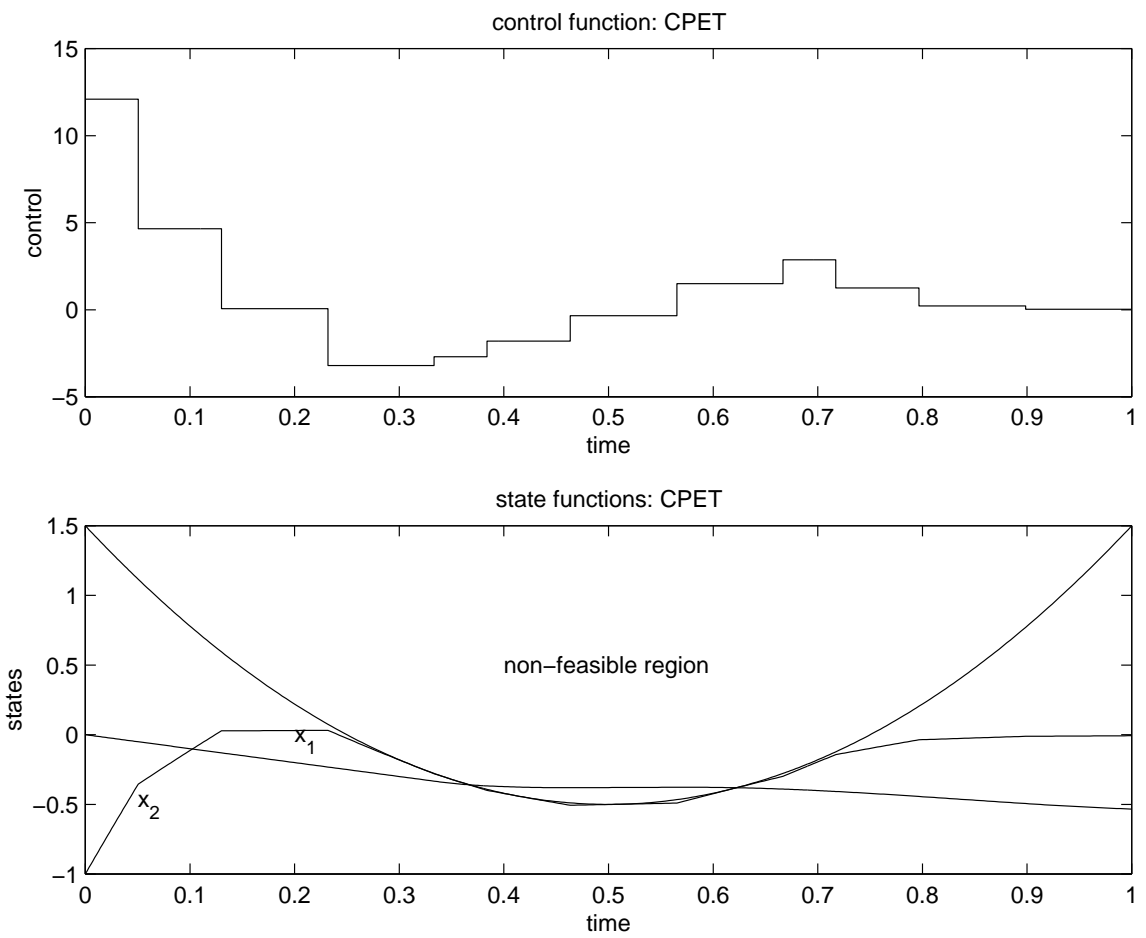

Figure 6: Example 5-Solution by CPET 


\section{References}

[1] L. S. Jennings, M. E. Fisher, K. L. Teo, and C. J. Goh. Miser3: Solving optimal control problems - an update. Advances in Engineering Software, 13(4):190-196, 1991. E158, E164

[2] K. Kaji and K. H. Wong. Nonlinearly constrained time-delayed optimal control problems. J. Optimization Theory and Applications, 82(2):295-313, 1994. E157, E169, E170, E177, E181

[3] H. W. J. Lee, K. L. Teo, L. S. Jennings, and V. Rehbock. Control parametrization enhancing technique for time optimal control problems. Dynamic Systems and Applications, 6(2):243-261, 1997. E156, E157

[4] H. W. J. Lee, K. L. Teo, Rehbock V, and L. S. Jennings. Control parametrization enhancing technique for optimal discrete-valued control problems. Automatica, 35(8):1401-1407, 1999. E156, E157

[5] K. Schittkowski. On the convergence of a sequential quadratic programming method with augmented lagrangian search function. Math. Operationsforschung und Statistik, Ser. Optimization, 14:197-216, 1993. E165

[6] K. L. Teo, C. J. Goh, and K. H. Wong. A Unified computational approach for optimal control problems. Longman Scientific and Technical, New York, 1991. E156, E158, E160, E161 
[7] K. L. Teo, L. S. Jennings, H. W. J. Lee, and V. Rehbock. Control parametrization enhancing technique for constrained optimal control problems. J. Austral. Math. Soc. B, 40(3):314-335, 1999. E156, E157, E168, E169

[8] K. L. Wong. A control parametrization algorithm for nonlinear time-lag optimal control problems. Opsearch, 25:177-184, 1988. E157, E161 